\title{
Electron ionization mass spectra of naphthoxazine, naphthpyrrolo- oxazinone and naphthoxazinobenzoxazine derivatives
}

\author{
Olli Martiskainen, ${ }^{1}$ Ferenc Fülöp, ${ }^{2}$ István Szatmári, ${ }^{2}$ and Kalevi Pihlaja ${ }^{1, *}$ \\ ${ }^{1}$ Structural Chemistry Group, Department of Chemistry, University of Turku, FI-20014 Turku, \\ Finland \\ ${ }^{2}$ Institute of Pharmaceutical Chemistry and Research Group for Stereochemistry, Hungarian \\ Academy of Sciences, University of Szeged, 6720 Eötvös u. 6, Szeged, Hungary \\ *Correspondence to: Prof. K. Pihlaja, Department of Chemistry, University of Turku, FI-20014 \\ Turku, Finland \\ E-mail:kpihlaja@utu.fi
}

Dedicated to Professor Harri Lönnberg on the occasion of his $60^{\text {th }}$ birthday

\begin{abstract}
Some naphthoxazinobenzoxazines (1a, 1b, 2a-2d, 3a, 3b), naphthoxazines $(\mathbf{4 a}, \mathbf{4 b}, \mathbf{5}, \mathbf{6 a}, \mathbf{6 b})$ and naphthpyrrolo-oxazinones $(\mathbf{7}, \mathbf{8 a}, \mathbf{8 b})$ were studied using mass spectrometry to find out regioisomeric effects and the effects of substituents. As expected the spectra of regioisomeric pairs 1a-3a $v s \mathbf{1 b}-\mathbf{3 b}$ were very different. For example the relative abundances of molecular ions were higher for 1a-3a and the $[\mathrm{M}-\mathrm{OH}]^{+}$ions were observed only for them. Compounds derived from (1- $\alpha$-aminobenzyl)-2-naphthol were usually characterized by abundant $m / z 231$ ions and 1-aminomethyl-2-naphthol derivatives by $\mathrm{m} / z 156$ ions. Ions related or similar to their complementary ions were also observed. Many of the studied compounds exhibited fairly abundant ions $\left[\mathrm{M}-\mathrm{C}_{17} \mathrm{H}_{11} \mathrm{O}_{2}\right]^{+}\left(\mathbf{1 a}, \mathbf{2 a}, 3 \mathbf{3 a}:[\mathrm{M}-247]^{+}\right),\left[\mathrm{M}-\mathrm{C}_{17} \mathrm{H}_{12} \mathrm{O}\right]^{+\bullet}\left(\mathbf{2 b}-\mathbf{2 d}:[\mathrm{M}-232]^{+\bullet}\right)$ or ion $\left[\mathrm{M}-\mathrm{C}_{11} \mathrm{H}_{8} \mathrm{O}\right]^{+\bullet}\left(\mathbf{1 b}, \mathbf{5}, \mathbf{6 a}, 7:[\mathrm{M}-156]^{+}\right)$. The results may be useful when making regiochemical conclusions.
\end{abstract}

Keywords: Electron ionization mass spectrometry, fused heterocyclic compounds, fragmentation pathways, regioisomers 


\section{Introduction}

Betti bases ${ }^{1}$ i.e. (1- $\alpha$-aminobenzyl)-2-naphthols, are useful synthons in the ring closure reactions. $^{2-4}$ They and their analogues commonly contain stereogenic centers and therefore regioselective and diastereoselective syntheses using Betti base synthons is gaining popularity. For example the ligands corresponding to the structure of $N, N$-dialkyl Betti base are becoming important in asymmetric syntheses catalyzed by metallic ions., ${ }^{5,6}$

(1- $\alpha$-Aminobenzyl)-2-naphthol and its derivatives can be transformed to naphthoxazinobenzoxazine derivatives via ring-closure reactions. ${ }^{3,4}$ Ring-closure reactions can be also used to synthesize naphthoxazinones and naphthoxazinoisoindolones from 1-aminomethyl-2-naphthol. ${ }^{3}$ These syntheses are usually highly diastereoselective. ${ }^{3,4}$

To continue our studies on the electron ionization mass spectrometry (EI MS) of condensed isomeric heterocycles, ${ }^{7,8}$ especially molecules containing 1,3-oxazine moiety, ${ }^{9-23}$ a group of naphthoxazine, naphthpyrrolo-oxazinone and naphthoxazino-benzoxazine derivatives were studied. The EI spectra of 16 compounds 1a, 1b, 2a-d, 3a, 3b, 4a, 4b, 5, 6a, 6b, 7, 8a, 8b, (the formulas are given in Chart 1 and the names ${ }^{3,4}$ in Table 1) were studied. Compounds consisted of three pairs of regioisomers $(\mathbf{1} \mathbf{a} / \mathbf{b}, \mathbf{2 a} / \mathbf{b}$ and $\mathbf{3 a} / \mathbf{b})$, the mass spectra of which were compared with each other. The other ten compounds were studied to clarify the effects of different substituents. The conformational analysis using NMR spectroscopy, molecular modelling and geometry optimization of selected compounds $\mathbf{2 b}, \mathbf{2 c}, \mathbf{8 b}$ and also 1a, $\mathbf{1 b}$, 3a were reported earlier. ${ }^{3,4}$ 
Table 1. The names of the compounds

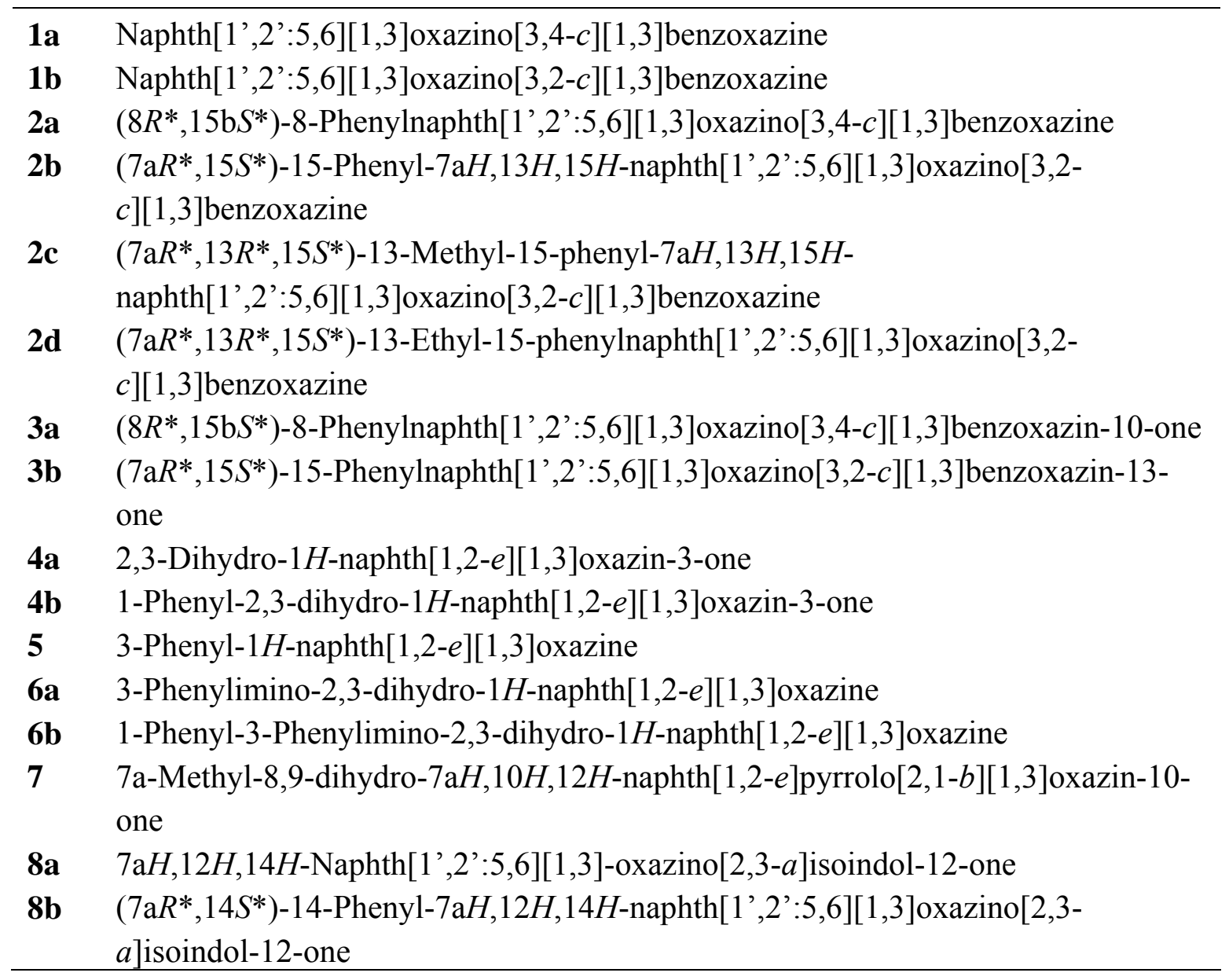


<smiles>c1ccc2c(c1)OCN1COc3ccc4ccccc4c3C21</smiles>

1a<smiles>CC1Oc2ccccc2[C@@H]2Oc3ccc4ccccc4c3[C@H](c3ccccc3)N12</smiles>

2c<smiles>O=C1NCc2c(ccc3ccccc23)O1</smiles>

$4 a$<smiles>c1ccc(/N=C2\NC(c3ccccc3)c3c(ccc4ccccc34)O2)cc1</smiles>

$6 b$<smiles>c1ccc2c(c1)OCN1Cc3c(ccc4ccccc34)OC21</smiles>

$1 b$<smiles>CC[C@H]1Oc2ccccc2[C@@H]2Oc3ccc4ccccc4c3[C@H](c3ccccc3)N21</smiles>

2d<smiles>O=C1NC(c2ccccc2)c2c(ccc3ccccc23)O1</smiles>

$4 b$<smiles>CC12CCC(=O)N1Cc1c(ccc3ccccc13)O2</smiles>

7<smiles>c1ccc([C@@H]2Oc3ccc4ccccc4c3[C@@H]3c4ccccc4OCN23)cc1</smiles>

2a<smiles>C[C@@]12c3ccccc3OC(=O)N1[C@@H](c1ccccc1)Oc1ccc3ccccc3c12</smiles>

$3 a$<smiles>c1ccc(C2=NCc3c(ccc4ccccc34)O2)cc1</smiles>

5<smiles>O=C1c2ccccc2[C@H]2Oc3ccc4ccccc4c3CN12</smiles>

$8 a$<smiles></smiles>

2b<smiles></smiles>

$3 \mathbf{b}$<smiles>c1ccc(/N=C2/NCc3c(ccc4ccccc34)O2)cc1</smiles>

$6 a$<smiles>O=C1c2ccccc2[C@H]2Oc3ccc4ccccc4c3[C@@H](c3ccccc3)N12</smiles>

$8 b$

Chart 1. The compounds studied.

The presence of 1,3-oxazine ring gives a possibility to ring-chain tautomeric equilibrium, this tautomerism being widely studied using NMR and MS methods. ${ }^{14-21}$ NMR studies of 1,3diarylnaphth[1,2-e][1,3]oxazines with similar structures to the compounds studied have been stated to show ring-chain tautomeric equilibria. ${ }^{23-25}$ The ring-openings related to tautomerism require a hydrogen-transfer. However in some ring-openings also a zwitterion can be formed. This does not require any hydrogen-transfer, for example photochromic compounds have been designed based on photoinduced ring opening of 1,3-oxazine ring. ${ }^{26,27}$ Ring opening has not been reported for the sixteen compounds studied now. The ring-chain tautomerism requiring transfer of hydrogen from nitrogen to oxygen is not possible for the studied molecules with tertiary 
nitrogen. However mass spectrometric processes involving ring opening have been reported also for 1,3-oxazine compounds with tertiary nitrogen. ${ }^{16}$

For 1,3-oxazines the most significant EI MS fragmentations can usually be interpreted based on the charge localisation on the nitrogen..$^{10,15,16,20,28}$ However the charge can also be localized on aromatic rings or oxygen, such is the case with octahydro-1,3-benzoxazines. ${ }^{20}$

Also the EI MS fragmentations of imino-compounds $6 \mathbf{a}$ and $\mathbf{6 b}$ can be compared with $N$ unsubstituted 4,5-cyclohexane-annelated 1,3-oxazines. ${ }^{29}$

\section{Experimental Section}

General Procedures. The electron ionization (EI) mass spectra were recorded on a VG ZABSpec mass spectrometer (VG Analytical, Division of Fisons, Manchester, UK), that was equipped with Opus V3.3X program package (Fisons Instruments, Manchester, UK). The ionization energy was $70 \mathrm{eV}$ and source temperature $160{ }^{\circ} \mathrm{C}$. Direct insertion probe was used. Perfluorokerosine (PFK) was used for calibration of the mass scale.

The fragmentation pathways were confirmed by B/E-linked scans for metastable ions. Also

$\mathrm{B}^{2} / \mathrm{E}$-linked scans were used to clarify these pathways. The low resolution, $\mathrm{B} / \mathrm{E}$ and $\mathrm{B}^{2} / \mathrm{E}$ spectra were measured using resolution of 3000. The accurate masses were determined by voltage scanning or by peak matching (10\% valley definition) using $6000-10000$ resolution for small $\mathrm{m} / \mathrm{z}$ values and over 10000 for the larger ones.

\section{Results and Discussion}

General fragmentation pathways are described in Scheme 1. Common fragment ions and their relative abundances $(R A)$ are listed in Tables 2 and 3 and other fragments in Table 4 and 5. Molecular ions $\left(\mathrm{M}^{+\bullet}\right)$ formed the base peaks of $\mathbf{2 a}, 7$ and $\mathbf{8 a}$. The ion, $m / z 128\left(\mathrm{C}_{10} \mathrm{H}_{8}{ }^{+\bullet}\right)$ was the base peak for $\mathbf{1 b}$ and $\mathbf{4 a}$. The base peak for 5 was the ion, $m / z 156\left(\mathrm{C}_{11} \mathrm{H}_{8} \mathrm{O}^{+\bullet}\right)$ and for $\mathbf{6 a}$ the ion, $m / z 157\left(\mathrm{C}_{11} \mathrm{H}_{9} \mathrm{O}^{+}\right)$. For compounds derived from (1- $\alpha$-aminobenzyl)-2-naphthol (1a, 2b-d, 3a, $3 \mathbf{b}, \mathbf{4 b}, \mathbf{6 b}, \mathbf{8 b}$ ), the base peak was always the ion $\mathrm{C}_{17} \mathrm{H}_{11} \mathrm{O}^{+}$at $\mathrm{m} / z$ 231. This ion was also observed for 2a, but with much lower $R A(27 \%)$. Due to the multiple bond cleavages required to form this ion $\mathrm{C}_{17} \mathrm{H}_{11} \mathrm{O}^{+}$, some new structures are to be formed, such as 1-(phenylmethylene)2(1H)-naphthalenonyl ion. Possible mechanism is presented in Scheme 2. The ion $\mathrm{C}_{17} \mathrm{H}_{11} \mathrm{O}^{+}$ fragments further by loosing $\mathrm{CO}$ or $\mathrm{HCO}$, resulting in ions $\mathrm{C}_{16} \mathrm{H}_{11}{ }^{+}(\mathrm{m} / z$ 203) of low abundance and $\mathrm{C}_{16} \mathrm{H}_{10}{ }^{+\bullet}$, respectively. The ion $\mathrm{C}_{16} \mathrm{H}_{10}{ }^{+\bullet}$ may have a structure resembling that of $(Z)-1$ ethynyl-2-(2-phenylethenyl)-benzene (Scheme 2). Also the ion $\mathrm{C}_{17} \mathrm{H}_{12} \mathrm{O}^{+\bullet}$ at $\mathrm{m} / z$ 232 was observed for $\mathbf{2 b}-\mathbf{2 d}, \mathbf{3 a}, \mathbf{3 b}, \mathbf{4 b}, \mathbf{6 b}, \mathbf{8 b}$. It can loose a hydrogen, $\mathrm{HCO}^{\bullet}$ or $\mathrm{CH}_{2} \mathrm{O}$. 
Table 2. Main electron induced fragmentations and their relative abundances $m / z(\% R A)$. Abundances are corrected for ${ }^{13} \mathrm{C}$ isotopes, 1b, 4a, 6a and 8a have been renormalised. Abundances are rounded to the nearest half percent. (Continued in table 3)

\begin{tabular}{lllll}
\hline Compound & $\mathrm{M}^{+\bullet}$ & {$[\mathrm{M}-\mathrm{H}]^{+}$} & {$[\mathrm{M}-\mathrm{OH}]^{+}$} & $\begin{array}{l}{[\mathrm{M}-\mathrm{CONH}]^{+}} \\
{\left[\mathrm{M}-\mathrm{CONH}_{2}\right]^{+}}\end{array}$ \\
\hline 1a & $289(82.5)$ & $288(32.5)$ & $272(33)$ & - \\
1b & $289(6)$ & - & - & - \\
2a & $365(100)$ & $364(4)$ & $348(67.5)$ & - \\
2b & $365(5.5)$ & - & - & - \\
2c & $379(3)$ & - & - & - \\
2d & $393(2.5)$ & - & - & - \\
3a & $379(36.5)$ & - & $362(4)$ & $336(9)$ \\
& & & & - \\
3b & $379(10.5)$ & - & - & - \\
4a & $199(46)$ & - & - & $156(96)$ \\
& & & & $155(4)$ \\
4b & $275(31)$ & - & - & $232(19)$ \\
& & & & - \\
$\mathbf{5}$ & $259(54)$ & $258(2)$ & - & - \\
6a & $274(68)$ & $273(32)$ & - & - \\
6b & $350(29)$ & $349(4.5)$ & - & - \\
7 & $253(100)$ & $252(1)$ & $236(33)$ & - \\
8a & $287(100)$ & $286(63)$ & $270(57.5)$ & - \\
8b & $363(48.5)$ & $362(4.5)$ & $346(8)$ & - \\
\hline
\end{tabular}

Also $[\mathrm{M}-\mathrm{Ph}]^{+}:$4b: 198(4), 6b: 273(44), 8b: 286(3) 
Table 3. More main electron induced fragmentations and their relative abundances $m / z(\% R A)$. Abundances are corrected for ${ }^{13} \mathrm{C}$ isotopes, 1b, 4a, 6a and 8a have been renormalised. Abundances are rounded to the nearest half percent

\begin{tabular}{|c|c|c|c|c|}
\hline Compound & $\mathrm{C}_{17} \mathrm{H}_{12} \mathrm{O}^{+\bullet}$ & $\mathrm{C}_{17} \mathrm{H}_{11} \mathrm{O}^{+}$ & $\begin{array}{l}\mathrm{C}_{11} \mathrm{H}_{9} \mathrm{O}^{+} \\
\mathrm{C}_{11} \mathrm{H}_{8} \mathrm{O}^{+}\end{array}$ & $\mathrm{C}_{10} \mathrm{H}_{8}^{+\bullet}$ \\
\hline 1a & $232(1)$ & $231(100)$ & - & $128(1.5)$ \\
\hline $\mathbf{1 b}$ & - & - & $\begin{array}{l}157(2.5) \\
156(51)\end{array}$ & $128(100)$ \\
\hline $2 a$ & $232(3)$ & $231(27)$ & - & - \\
\hline $2 b$ & $232(23)$ & $231(100)$ & - & - \\
\hline 2c & $232(22)$ & $231(100)$ & - & - \\
\hline 2d & $232(24)$ & $231(100)$ & - & - \\
\hline 3a & $232(12)$ & $231(100)$ & - & - \\
\hline $3 \mathbf{b}$ & $232(24)$ & $231(100)$ & - & - \\
\hline $4 a$ & - & - & $\begin{array}{l}157(4) \\
156(96)\end{array}$ & $128(100)$ \\
\hline $4 b$ & $232(19)$ & $231(100)$ & - & - \\
\hline 5 & - & - & $\begin{array}{l}- \\
156(100)\end{array}$ & $128(70)$ \\
\hline $6 a$ & - & - & $\begin{array}{l}157(100) \\
156(40)\end{array}$ & $128(76)$ \\
\hline $6 b$ & $232(16)$ & $231(100)$ & - & $128(2)$ \\
\hline 7 & - & - & $\begin{array}{l}157(71) \\
156(72)\end{array}$ & $128(95)$ \\
\hline $8 a$ & - & $231(2)$ & $156(26.5)$ & $128(60)$ \\
\hline $8 b$ & $232(14)$ & $231(100)$ & & $128(0.5)$ \\
\hline
\end{tabular}


Table 4. Other fragments (relative abundances mostly $>3 \%$ ) for compounds $1 \mathbf{a}, \mathbf{1 b}, \mathbf{2 a}-\mathbf{d}, \mathbf{3 a}$, $3 \mathbf{b}, \mathbf{4 a}, \mathbf{4 b}, \mathbf{5} . R A$ s are corrected for ${ }^{13} \mathrm{C}$ isotopes. $\mathbf{1 b}$ and $\mathbf{4 a}$ have been renormalised. Abundances are rounded to the nearest half percent

\begin{tabular}{|c|c|}
\hline Compound & $m / z(\% R A)$ \\
\hline 1a & $\begin{array}{l}\text { 260: } \mathrm{C}_{18} \mathrm{H}_{12} \mathrm{O}_{2}^{+\bullet}(5)+\mathrm{C}_{18} \mathrm{H}_{14} \mathrm{NO}^{+}(1), \mathrm{C}_{17} \mathrm{H}_{11} \mathrm{O}_{2}^{+}: 247(22), 219(2), \mathrm{C}_{16} \mathrm{H}_{10} \mathrm{O}^{+\bullet}: \\
218(11), \mathrm{C}_{16} \mathrm{H}_{11}{ }^{+}: 203(2), \mathrm{C}_{16} \mathrm{H}_{10}{ }^{+\bullet}: 202(8), \mathrm{C}_{16} \mathrm{H}_{8}^{+\bullet}: 200(3), \mathrm{C}_{15} \mathrm{H}_{11}{ }^{+}: 191(4), \\
\mathrm{C}_{15} \mathrm{H}_{9}^{+}: 189(19), \mathrm{C}_{13} \mathrm{H}_{9}^{+}: 165(5), \mathrm{C}_{13} \mathrm{H}_{8}^{+}: 164(2), \mathrm{C}_{13} \mathrm{H}_{7}^{+}: 163(5), 139(4), 101(3), \\
42(78)\end{array}$ \\
\hline 1b & $\begin{array}{l}\mathrm{C}_{8} \mathrm{H}_{7} \mathrm{NO}^{+\bullet}: 133(52), \mathrm{C}_{8} \mathrm{H}_{6} \mathrm{NO}^{+}: 132(25), \mathrm{C}_{10} \mathrm{H}_{7}^{+}: 127(16), 126(8), 106(1.5), 105: \\
\mathrm{C}_{7} \mathrm{H}_{7} \mathrm{~N}^{+}(8)+\mathrm{C}_{7} \mathrm{H}_{5} \mathrm{O}^{+}(13), \mathrm{C}_{7} \mathrm{H}_{6} \mathrm{~N}^{+}: 104(14), 103(1), \mathrm{C}_{8} \mathrm{H}_{6}^{+\bullet}: 102(12), \mathrm{C}_{8} \mathrm{H}_{5}^{+}: \\
101(4), 78(32), 77(20), 76(9), 75(10), 74(11), 69(4), 64(7), 63(13), 62(6), 52(9), \\
\mathrm{C}_{4} \mathrm{H}_{3}{ }^{+}: 51(32), 50(19), 44(3)\end{array}$ \\
\hline 2a & $\begin{array}{l}\mathrm{C}_{18} \mathrm{H}_{12} \mathrm{O}_{2}^{+\bullet}: 260(12), \mathrm{C}_{17} \mathrm{H}_{11} \mathrm{O}_{2}^{+}: 247(6), 218(6), \mathrm{C}_{16} \mathrm{H}_{10}{ }^{+\bullet}: 202(8), \mathrm{C}_{15} \mathrm{H}_{9}^{+}: 189(6), \\
\mathrm{C}_{8} \mathrm{H}_{8} \mathrm{~N}^{+}: 118(77), 117(3), \mathrm{C}_{7} \mathrm{H}_{7}^{+}: 91(44)\end{array}$ \\
\hline 2b & $\begin{array}{l}203(3), \mathrm{C}_{16} \mathrm{H}_{10}{ }^{+\bullet}: 202(21), \mathrm{C}_{16} \mathrm{H}_{9}^{+}: 201(2), \mathrm{C}_{16} \mathrm{H}_{8}^{+\bullet}: 200(4), \mathrm{C}_{8} \mathrm{H}_{7} \mathrm{NO}^{+\bullet}: 133(14), \\
\mathrm{C}_{8} \mathrm{H}_{6} \mathrm{NO}^{+}: 132(7), 115.4(\text { metastable, 4), 105(5), 104(4), 101(5), 78(8), 77(6), 76(3), } \\
\text { 51(7), 50(4), 45(4) }\end{array}$ \\
\hline 2c & $\begin{array}{l}\mathrm{C}_{26} \mathrm{H}_{21} \mathrm{NO}^{+\bullet} \text { (impurity): 363(4), 203(3), } \mathrm{C}_{16} \mathrm{H}_{10}^{+\bullet}: \text { 202(22), 201(2), 200(4), } \\
\mathrm{C}_{9} \mathrm{H}_{9} \mathrm{NO}^{+\bullet}: 147(11), 146(5), \mathrm{C}_{8} \mathrm{H}_{6} \mathrm{NO}^{+}: 132(26), 120(4), 115.4(\text { metastable, 4), } \\
\mathrm{C}_{8} \mathrm{H}_{5}^{+}: 101(5), 91(4), 78(3), 77(5), 76(3), 63(3), 51(8), 50(4)\end{array}$ \\
\hline 2d & $\begin{array}{l}\text { 203(3), 202(22), 201(2), 200(4), } \mathrm{C}_{10} \mathrm{H}_{11} \mathrm{NO}^{+*}: 161(6), \mathrm{C}_{9} \mathrm{H}_{8} \mathrm{NO}^{+}: 146(4), \mathrm{C}_{8} \mathrm{H}_{6} \mathrm{NO}^{+}: \\
132(52), 120(4), 116(3), 15.4(4), 101(5), 78(3), 77(7), 76(3), 63(3), 51(10), 50(4)\end{array}$ \\
\hline 3a & $\begin{array}{l}363(5), \mathrm{C}_{17} \mathrm{H}_{11} \mathrm{O}_{2}^{+}: 247(13), \mathrm{C}_{16} \mathrm{H}_{10} \mathrm{O}^{+\bullet}: 218(7), \mathrm{C}_{16} \mathrm{H}_{10}{ }^{+}: 202(9), 191(4), \mathrm{C}_{15} \mathrm{H}_{9}{ }^{+}: \\
189(10), \mathrm{C}_{8} \mathrm{H}_{6} \mathrm{NO}^{+}: 132(52), \mathrm{C}_{7} \mathrm{H}_{5} \mathrm{O}^{+}: 105(5), 104: \mathrm{C}_{7} \mathrm{H}_{6} \mathrm{~N}^{+}(3)+\mathrm{C}_{7} \mathrm{H}_{4} \mathrm{O}^{+}(1), 77(18), \\
\text { 51(4), 44(5), 40(7) }\end{array}$ \\
\hline $\mathbf{3 b}$ & $203(3), \mathrm{C}_{16} \mathrm{H}_{10}{ }^{+\bullet}: 202(15), \mathrm{C}_{7} \mathrm{H}_{5} \mathrm{NO}^{+\bullet}: 119(2)$ \\
\hline 4a & $\begin{array}{l}272 \text { (impurity, 3), } 231 \text { (impurity, 3), } \mathrm{C}_{11} \mathrm{H}_{11} \mathrm{NO}^{+\bullet} \text { (reactant, impurity): 173(12), } \\
170(3), 160(3), \mathrm{C}_{11} \mathrm{H}_{9} \mathrm{O}^{+}: 157(4), 129(3), \mathrm{C}_{10} \mathrm{H}_{7}^{+}: 127(9), \mathrm{C}_{10} \mathrm{H}_{6}^{+}:: 126(7), \mathrm{C}_{9} \mathrm{H}_{7}^{+}: \\
115(9.5), \mathrm{C}_{9} \mathrm{H}_{6}^{+\bullet}: 114(3.5), \mathrm{C}_{8} \mathrm{H}_{6}^{+}: 102(7), 101(4), 100(3.5), 78(3), 77(6), 76(4), \\
75(4), 74(5), 72(3), 64(5), 63(8), 58(4), 51(8)\end{array}$ \\
\hline 4b & $\mathrm{C}_{16} \mathrm{H}_{11}^{+}: 203(3), \mathrm{C}_{16} \mathrm{H}_{10}{ }^{+}: 202(14), 116(5), \mathrm{C}_{9} \mathrm{H}_{7}^{+}: 115(8), \mathrm{C}_{8} \mathrm{H}_{5}^{+}: 101(5)$ \\
\hline 5 & $\begin{array}{l}\text { 129.7(4), 129(2), 127(14), 126(5), } \mathrm{C}_{7} \mathrm{H}_{5} \mathrm{~N}^{+\bullet}: 103(5), \mathrm{C}_{8} \mathrm{H}_{6}^{+*}: 102(7), \mathrm{C}_{6} \mathrm{H}_{5}^{+}: \text {77(6), } \\
76(4)\end{array}$ \\
\hline
\end{tabular}


Table 5. Other fragments (relative abundances mostly $>3 \%$ ) of $\mathbf{6 a}, \mathbf{6 b}, \mathbf{7}, \mathbf{8 a}, \mathbf{8 b} . R A$ s are corrected for ${ }^{13} \mathrm{C}$ isotopes. 6a and $\mathbf{8 a}$ have been renormalised. Abundances are rounded to the nearest half percent

\begin{tabular}{ll}
\hline Compound & $m / z(\% R A)$ \\
\hline 6a & $\mathrm{C}_{12} \mathrm{H}_{8} \mathrm{NO}^{+}: 182(11), 137(8), 129(6), 127(18), 126(5), \mathrm{C}_{7} \mathrm{H}_{6} \mathrm{~N}_{2}{ }^{+\bullet}: 118(15), \mathrm{C}_{9} \mathrm{H}_{7}^{+}:$ \\
& $115(3), \mathrm{C}_{8} \mathrm{H}_{6}^{+\bullet}: 102(6), 91(5), 77(11), 51(5)$ \\
$\mathbf{6 b}$ & $\mathrm{C}_{18} \mathrm{H}_{12} \mathrm{NO}^{+}: 258(3), 233: \mathrm{C}_{17} \mathrm{H}_{13} \mathrm{O}^{+}(9), 203(3), \mathrm{C}_{16} \mathrm{H}_{10}{ }^{+\bullet}: 202(15), 175(4), 136(4)$, \\
& $127(3), \mathrm{C}_{7} \mathrm{H}_{6} \mathrm{~N}_{2}^{+\bullet}: 118(2), 77(3)$ \\
7 & $\mathrm{C}_{15} \mathrm{H}_{12} \mathrm{NO}_{2}{ }^{+}: 238(8), \mathrm{C}_{15} \mathrm{H}_{15} \mathrm{NO}: 225(1.5), \mathrm{C}_{15} \mathrm{H}_{14} \mathrm{NO}: 224(2), 182:$ \\
& $\mathrm{C}_{13} \mathrm{H}_{12} \mathrm{~N}^{+}(1)+\mathrm{C}_{12} \mathrm{H}_{8} \mathrm{NO}^{+}(2), \mathrm{C}_{13} \mathrm{H}_{9} \mathrm{O}^{+}: 181(1.5), \mathrm{C}_{12} \mathrm{H}_{8} \mathrm{O}^{+\bullet}: 168(5), 158(2), 155(4)$, \\
& $129(5), 127(25), 126(6), 115(4), 102(8), 101(3), 98.7(3), \mathrm{C}_{5} \mathrm{H}_{7} \mathrm{NO}^{+}: 97(28), 78(3)$, \\
& $77(5), 69(4), 64(3), 51(3), 42(4)$ \\
8a & $286(63), \mathrm{C}_{18} \mathrm{H}_{12} \mathrm{NO}^{+}: 258(4), \mathrm{C}_{17} \mathrm{H}_{12} \mathrm{~N}^{+}: 230(7.5), 143.5(5), 130(3), 127(10), 102:$ \\
& $\mathrm{C}_{8} \mathrm{H}_{6}^{+\bullet}(4.5)+\mathrm{C}_{7} \mathrm{H}_{4} \mathrm{~N}^{+}(1), 77(3.5), 76(6)$ \\
8b & $203(3), \mathrm{C}_{16} \mathrm{H}_{10}{ }^{+}: 202(19), 40(3)$ \\
&
\end{tabular}

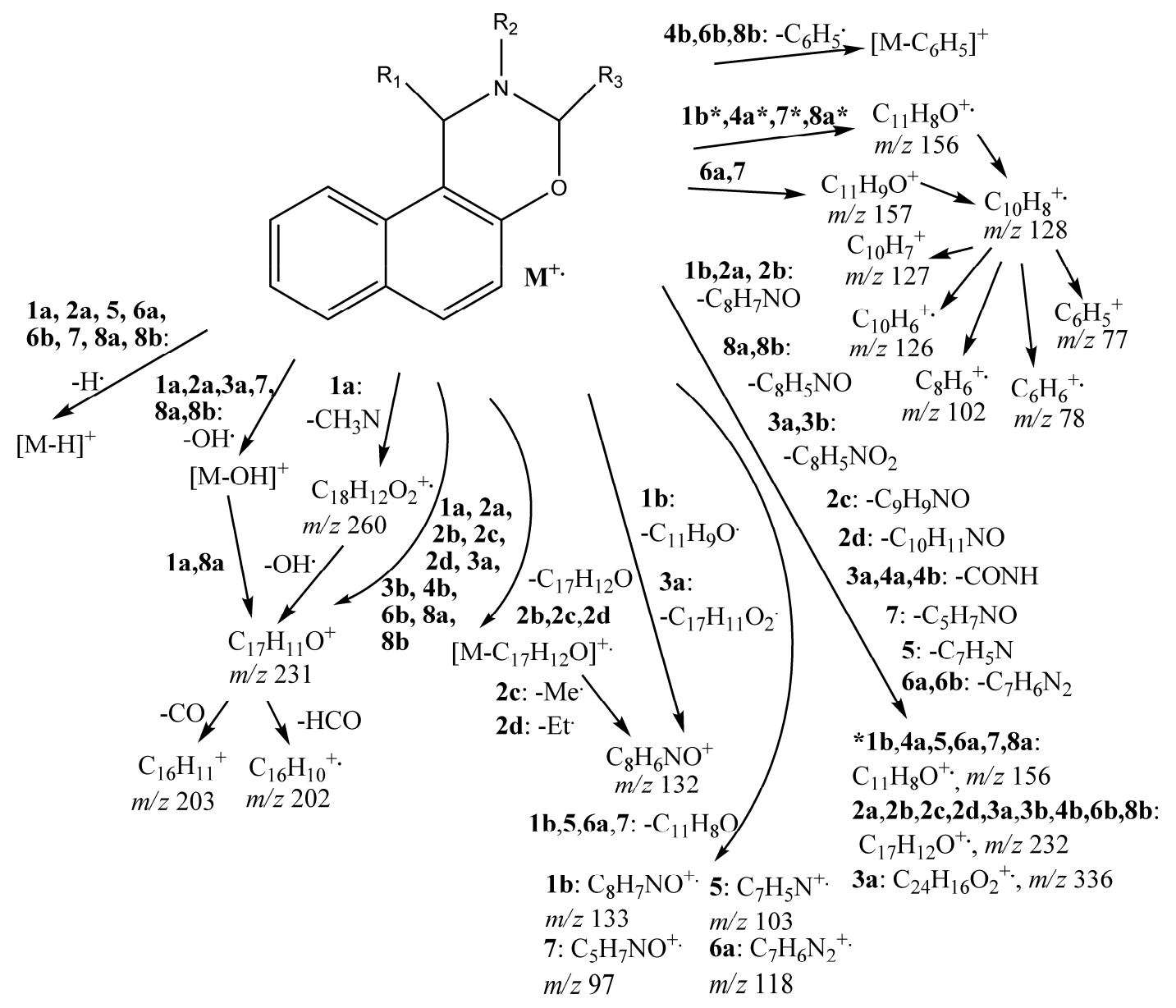

Scheme 1 


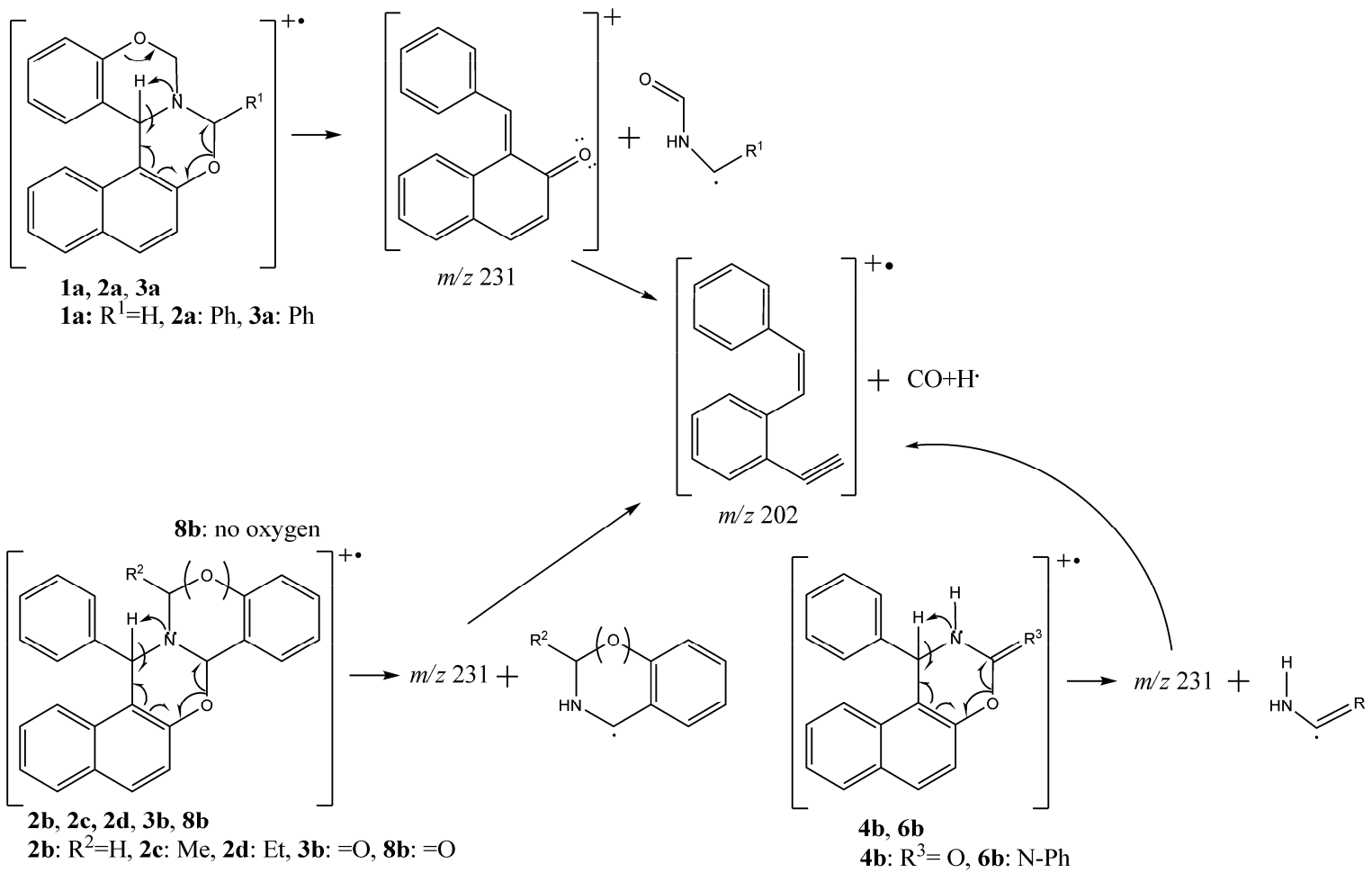

Scheme 2. Suggested mechanism for the formation of ions $m / z 231$ and $m / z 202$.

The ion $\mathrm{C}_{11} \mathrm{H}_{8} \mathrm{O}^{+\bullet}$ at $m / z 156$ was observed for compounds $\mathbf{1 b}, \mathbf{4 a}, \mathbf{5}, \mathbf{6 a}, \mathbf{7}, \mathbf{8 a}$, i.e. for compounds derived from 1-aminomethyl-2-naphthol. This ion is probably formed via mechanism similar to that presented for the formation of ion $m / z 231$. The ion $m / z 156$ looses also $\mathrm{CO}$ or $\mathrm{HCO}$, resulting in ions $\mathrm{C}_{10} \mathrm{H}_{8}{ }^{+\bullet}$ or $\mathrm{C}_{10} \mathrm{H}_{7}{ }^{+}$. An abundant ion $\mathrm{C}_{11} \mathrm{H}_{9} \mathrm{O}^{+}$at $m / z 157$ was observed also for $\mathbf{6 a}$ and $\mathbf{7}$ and a weak one for $\mathbf{4 a}$.

In the case of 7 ion $\mathrm{C}_{11} \mathrm{H}_{9} \mathrm{O}^{+}$is formed by loss of $\mathrm{C}_{5} \mathrm{H}_{6} \mathrm{NO}^{\circ}$ from the molecular ion or via the route $\mathrm{M}^{+} \cdot-\mathrm{OH}^{\bullet}-\mathrm{C}_{5} \mathrm{H}_{5} \mathrm{~N}$, both of these routes requiring $\mathrm{H}$-transfer. The ion $\mathrm{C}_{11} \mathrm{H}_{9} \mathrm{O}^{+}$in turn looses $\mathrm{CO}$ or $\mathrm{COH}^{\bullet}$, and forms the ions $\mathrm{C}_{8} \mathrm{H}_{6}^{+\bullet}$ and $\mathrm{C}_{6} \mathrm{H}_{5}{ }^{+}$.

The ion $[\mathrm{M}-\mathrm{OH}]^{+}$was most abundant for 2a $(R A 67.5 \%)$ and $\mathbf{8 a}(R A 57.5 \%)$. It was also formed by compounds 1a, 3a, 7, and $\mathbf{8 b}$. It is questionable which oxygen is lost with the hydroxyl. OH-loss requires hydrogen migration after ionization and ring-opening, possible mechanisms involving distonic ions shown in Scheme 3. The OH-loss from 3a having a carbonyl group cannot proceed via the first route in Scheme 3; based on steric reasons the carbonyl oxygen may abstract hydrogen from the position bearing the phenyl substituent. Solving the exact mechanism would require complicated deuterium labeling. For 2a the B/E scans of the [M $-\mathrm{OH}]^{+}$ion gave no significant signals, so this ion is unusually stable, the positive charge being probably stabilized by the aromatic groups. For 1a the ion $[\mathrm{M}-\mathrm{OH}]^{+}$may fragment further by loss of $\mathrm{C}_{2} \mathrm{H}_{3} \mathrm{~N}$ forming the ion $m / z 231$, and $\mathrm{B}^{2} / \mathrm{E}$ scan revealed also the $\mathrm{C}_{3} \mathrm{H}_{4} \mathrm{~N}^{\bullet}$ loss. If the ion at $\mathrm{m} / \mathrm{z} 231$ from 1a has the structure described in Scheme 2, then the oxygen that is attached to the phenyl group is the one lost with $\mathrm{OH}$ and not the oxygen attached to the naphthyl group. Also 
based on the geometry ${ }^{4}$ the phenyl oxygen is more likely to be lost as hydroxyl. $[\mathrm{M}-\mathrm{OH}]^{+}$from 7 fragments further by loss of $\mathrm{C}_{3} \mathrm{H}_{5} \mathrm{~N}\left(\mathrm{C}_{13} \mathrm{H}_{9} \mathrm{O}^{+}, m / z\right.$ 181), $\mathrm{C}_{5} \mathrm{H}_{5} \mathrm{~N}\left(\mathrm{C}_{11} \mathrm{H}_{9} \mathrm{O}^{+}, m / z\right.$ 157) or $\mathrm{C}_{11} \mathrm{H}_{7}$ $\left(\mathrm{C}_{5} \mathrm{H}_{7} \mathrm{O}^{+}, m / z\right.$ 97) and that from $\mathbf{8 b}$ by loss of $\mathrm{C}_{8} \mathrm{H}_{5} \mathrm{~N}\left(\mathrm{C}_{17} \mathrm{H}_{11} \mathrm{O}^{+}, m / z 231\right)$. For $\mathbf{7}$ and $\mathbf{8 b}$ (and perhaps also for 8a) it is probable that the hydroxyl group is formed from the carbonyl group by hydrogen migration. In this case the naphthyl oxygen is not lost as hydroxyl.

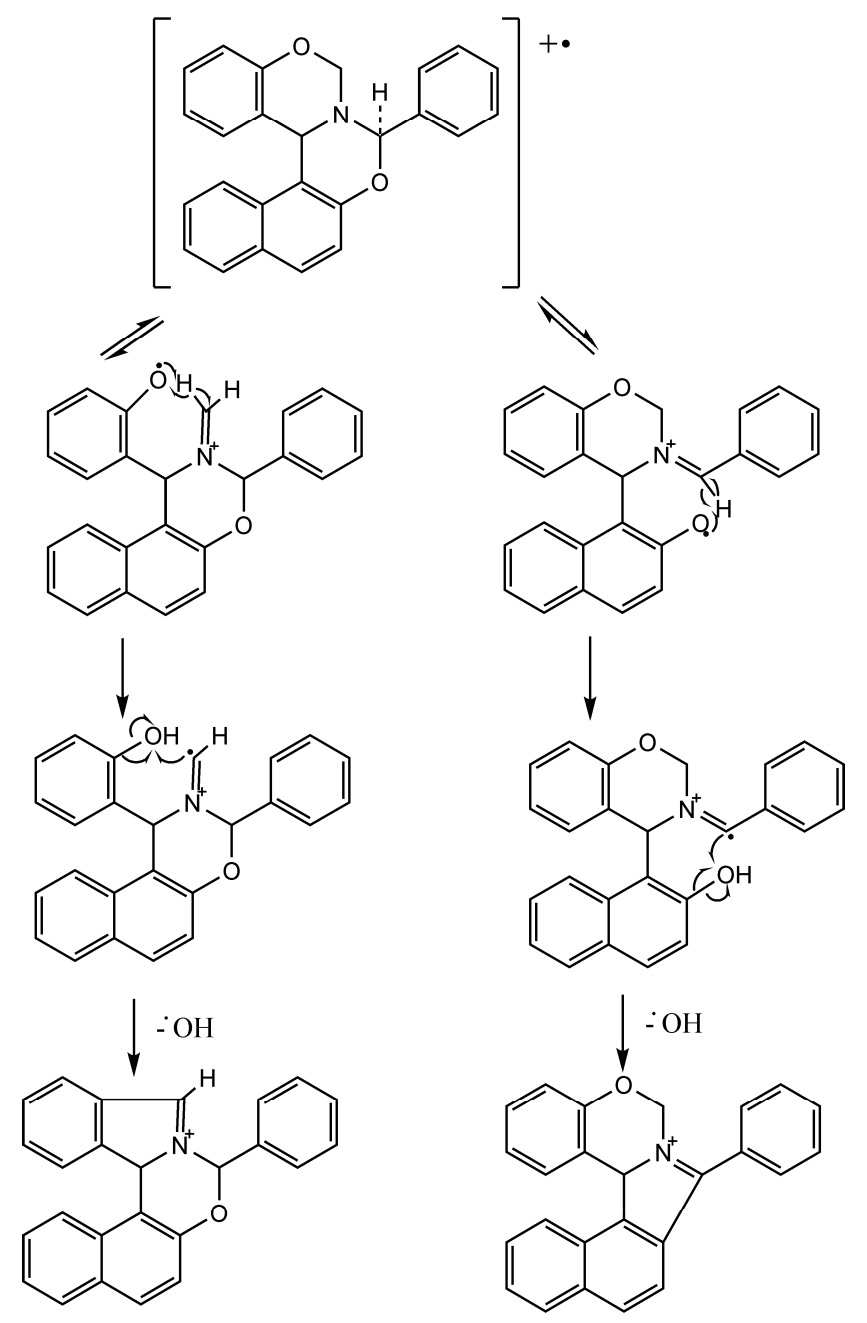

Scheme 3. Possible ring openings of $2 \mathrm{a}$ and the consequent $\mathrm{OH}-\mathrm{loss}$.

Weak CO loss was observed only for $\mathbf{7}$ and $\mathbf{8 a} \cdot[\mathrm{M}-\mathrm{CONH}]^{+\bullet}$ ion was abundant for $\mathbf{4 a}(R A$ 94\%) and was also present for $\mathbf{3 a}$ and $\mathbf{4 b}$. An abundant $[\mathrm{M}-\mathrm{Ph}]^{+}$ion $(R A 44 \%)$ was exhibited by $\mathbf{6 b}$, and traces by $\mathbf{4} \mathbf{b}$ and $\mathbf{8 b}$. The phenyl substituent is axial in $\mathbf{2} \mathbf{b}-\mathbf{d},{ }^{4}$ and hence they are expected to fragment similarly but different from $\mathbf{2 a}$ in which the phenyl group is equatorial. ${ }^{4}$ This may at least partly explain why 2a gives an abundant $\mathrm{C}_{7} \mathrm{H}_{7}{ }^{+}$ion, but $\mathbf{2 b}-\mathbf{d}$ not. In contrast to 2a for which the base peak is that of the molecular ion, compounds $\mathbf{2 b}-\mathbf{d}$ all exhibit $\mathrm{M}^{+\bullet}$ ions of low abundance and the ions $\mathrm{C}_{17} \mathrm{H}_{11} \mathrm{O}^{+}(\mathrm{m} / z$ 231) form their base peaks instead. Common ions of 
similar abundance for $\mathbf{2 b}-\mathbf{d}$ are $\mathrm{C}_{17} \mathrm{H}_{12} \mathrm{O}^{+\bullet}$ at $\mathrm{m} / z 232$ and $\mathrm{C}_{16} \mathrm{H}_{10}{ }^{+\bullet}$ at $m / z$ 202. Also a metastable transition corresponding to $\mathrm{C}_{17} \mathrm{H}_{11} \mathrm{O}^{2+}$ was observed.

The methyl- (2c: $\mathrm{R}=\mathrm{Me}$ ) and ethyl-substituted (2d: $\mathrm{R}=\mathrm{Et})$ gave very similar spectra. In addition to the common ions mentioned before, both have nearly identical spectra below $\mathrm{m} / \mathrm{z} 120$. The greatest differences are as follows: the ion $m / z 147\left(\mathrm{C}_{9} \mathrm{H}_{9} \mathrm{NO}^{+\bullet}\right)$ was obtained only for 2c and the ion $m / z 161\left(\mathrm{C}_{10} \mathrm{H}_{11} \mathrm{NO}^{+\bullet}\right)$ for 2d. Furthermore the ion $m / z 132\left(\mathrm{C}_{8} \mathrm{H}_{6} \mathrm{NO}^{+}\right)$was most abundant for 2d. When the molecular ions loose $\mathrm{C}_{17} \mathrm{H}_{12} \mathrm{O}$, the ions $\mathrm{C}_{10} \mathrm{H}_{11} \mathrm{NO}^{+\bullet}$ (2d, $m / z$ 161), $\mathrm{C}_{9} \mathrm{H}_{9} \mathrm{NO}^{+\bullet}\left(2 \mathbf{c}, m / z\right.$ 147) and $\mathrm{C}_{8} \mathrm{H}_{7} \mathrm{NO}^{+\bullet}(2 \mathbf{b}, m / z$ 133) are formed. Similar fragment ions have been observed for substituted 1,3-benzoxazino[4,3-b][1,3]1,3-benzoxazines ${ }^{16}$, where the bond between $\mathrm{C}-7$ and $\mathrm{O}$ is cleaved first, resulting to ring-opening of the oxazine ring.

For 2c and 2d the ion $\mathrm{C}_{8} \mathrm{H}_{6} \mathrm{NO}^{+}\left(m / z\right.$ 132) is formed via routes $\mathrm{C}_{9} \mathrm{H}_{9} \mathrm{NO}^{+\bullet}-\mathrm{Me}^{\bullet}(\mathbf{2 c})$ and $\mathrm{C}_{10} \mathrm{H}_{11} \mathrm{NO}^{+\bullet}-\mathrm{Et}^{\bullet}(\mathbf{2 d})$. For the other two compounds (1b and 3a) without an alkyl substituent at C-2 or C-2' the ion $m / z 132$ is formed directly from the molecular ion instead.

The spectra of $\mathbf{4 a}(\mathrm{R}=\mathrm{H})$ and $\mathbf{4 b}(\mathrm{R}=\mathrm{Ph})$ differ greatly from each other. For $\mathbf{4 b}$ the ion $[\mathrm{M}$ $\left.-\mathrm{CONH}_{2}\right]^{+}$, i.e. $\mathrm{C}_{17} \mathrm{H}_{11} \mathrm{O}^{+}$, forms the base peak whereas for $\mathbf{4 a}$ the base peak locates at $\mathrm{m} / z 128$ $\left(\mathrm{C}_{10} \mathrm{H}_{8}^{+\bullet}\right)$ and it also shows a strong $[\mathrm{M}-\mathrm{CONH}]^{+\bullet}$ ion. The same is true for $\mathbf{6 a}(\mathrm{R}=\mathrm{H})$ and $\mathbf{6 b}$ $(\mathrm{R}=\mathrm{Ph})$ : $6 \mathbf{b}$ exhibits an abundant ion at $m / z 231, \mathrm{C}_{17} \mathrm{H}_{11} \mathrm{O}^{+}$; based on $\mathrm{B}^{2} / \mathrm{E}$ scans it is formed partly via route $\mathrm{M}^{+\bullet}-\mathrm{NHPh}{ }^{\bullet}-\mathrm{HCN}$ and partly via route $\mathrm{M}^{+\bullet}-\mathrm{Ph}^{\bullet}-\mathrm{CH}_{2} \mathrm{~N}_{2}$. 6 a in turn shows an abundant $[\mathrm{M}-\mathrm{NCNPh}]^{+}$ion, $\mathrm{C}_{11} \mathrm{H}_{9} \mathrm{O}^{+}$, at $m / z 157$ and a moderately abundant ion, $\mathrm{C}_{11} \mathrm{H}_{8} \mathrm{O}^{+\bullet}$, at $m / z$ 156; based on $\mathrm{B}^{2} / \mathrm{E}$ scans also routes $\mathrm{M}^{+\bullet}-\mathrm{NHPh}^{\bullet}-\mathrm{CN}^{\bullet}$ and $\mathrm{M}^{+\bullet}-\mathrm{Ph}^{\bullet}-\mathrm{NHCN}^{\bullet}$ are possible. 6a exhibits a strong ion at $m / z 128, \mathrm{C}_{10} \mathrm{H}_{8}{ }^{+\bullet}$, but this is very weak for $6 \mathbf{b}$. For $6 \mathbf{b}$ [M $\mathrm{Ph}]^{+}$can be observed, but 6 a exhibits relatively abundant $[\mathrm{M}-\mathrm{H}]^{+}$ion - so it is probable that the $\mathrm{Ph}-\mathrm{C}$ rather than the $\mathrm{Ph}-\mathrm{N}$ bond is cleaved. Both $6 \mathbf{6}$ and $\mathbf{6 b}$ gave also weak $[\mathrm{M}-\mathrm{NHPh}]^{+}$ions $(R A<10 \%)$.

In addition to the ion $m / z 231, \mathrm{C}_{17} \mathrm{H}_{11} \mathrm{O}^{+}$or ion $m / z 156, \mathrm{C}_{11} \mathrm{H}_{8} \mathrm{O}^{+\bullet}$ compounds 1a, 2a-d, 3a, $\mathbf{3 b}, \mathbf{4 b}, \mathbf{6 b}$ and $\mathbf{8 b}$ also show ions related or similar to their complementary ion. The ion [M $\left.\mathrm{C}_{17} \mathrm{H}_{11} \mathrm{O}_{2}\right]^{+}$was abundant for $\mathbf{1 a}-3 \mathbf{a}$. The resulting ions were $\mathrm{C}_{2} \mathrm{H}_{4} \mathrm{~N}^{+}(\mathbf{1 a}), \mathrm{C}_{8} \mathrm{H}_{8} \mathrm{~N}^{+}$(2a) and $\mathrm{C}_{8} \mathrm{H}_{6} \mathrm{NO}^{+}$(3a). The ions corresponding to $[\mathrm{M}-232]^{+\bullet}$, i.e. $\mathrm{C}_{8} \mathrm{H}_{7} \mathrm{NO}^{+\bullet}(\mathbf{2 b}), \mathrm{C}_{9} \mathrm{H}_{9} \mathrm{NO}^{+\bullet}$ (2c) and $\mathrm{C}_{10} \mathrm{H}_{11} \mathrm{NO}^{+\bullet}$ (2d), were moderately abundant. Also $6 \mathbf{b}$ exhibited a weak $[\mathrm{M}-232]^{+\bullet}$, i.e. $\mathrm{C}_{7} \mathrm{H}_{6} \mathrm{~N}_{2}{ }^{+\bullet}$ ion. $[\mathrm{M}-156]^{+\bullet}$, i.e. $\left[\mathrm{M}-\mathrm{C}_{11} \mathrm{H}_{8} \mathrm{O}\right]^{+\bullet}$ was also observed for $\mathbf{1 b}, \mathbf{5}, \mathbf{6 a}$ and 7: a very abundant $\mathrm{C}_{8} \mathrm{H}_{7} \mathrm{NO}^{+\bullet}$ ion for $\mathbf{1 b}$, a rather abundant $\mathrm{C}_{5} \mathrm{H}_{7} \mathrm{NO}^{+\bullet}$ ion for $\mathbf{7}$ and moderately abundant $\mathrm{C}_{7} \mathrm{H}_{5} \mathrm{~N}^{+\bullet}$ ion for 5 and similarly $\mathrm{C}_{7} \mathrm{H}_{6} \mathrm{~N}_{2}{ }^{+\bullet}$ ion for $\mathbf{6 a}$. For $\mathbf{3 b}, \mathbf{4 b}$, and $\mathbf{8 b}$ only traces of [M $232]^{+\bullet}$ could be observed, otherwise these compounds and $\mathbf{4 a}, \mathbf{6 b}$ and $\mathbf{8 a}$ did not show ions at $\mathrm{m} / \mathrm{z}$ $(\mathrm{M}-247)$ and $(\mathrm{M}-156)$. The carbonyl group in $3 \mathbf{b}$ seems to prevent the formation of $[\mathrm{M}-$ $\left.\mathrm{C}_{17} \mathrm{H}_{12} \mathrm{O}\right]^{+\bullet}$ in contrast to structurally similar $\mathbf{2 b}-\mathbf{d}$ with alkyl or hydrogen at $\mathrm{C}-13$.

The formation of $\mathrm{C}_{11} \mathrm{H}_{9} \mathrm{O}^{+}$from the molecular ion of $\mathbf{6 a}$ is probably initiated by an $\mathrm{H}$-transfer from either $\mathrm{NH}$ or phenyl group to oxygen and a subsequent $\operatorname{loss}$ of $\mathrm{C}_{7} \mathrm{H}_{5} \mathrm{~N}_{2}$. This fragmentation is different from that found for $N$-unsubstituted 4,5-cyclopentane-fused 1,3-oxazines ${ }^{29}$ where $\mathrm{C}_{7} \mathrm{H}_{5} \mathrm{NO}^{\cdot}$ but no $\mathrm{C}_{7} \mathrm{H}_{5} \mathrm{~N}_{2}{ }^{\circ}$ loss was observed. This may be caused by different annelation 
positions. Another difference is the ion $\left[\mathrm{M}-\mathrm{C}_{6} \mathrm{H}_{6} \mathrm{~N}\right]^{+}(\mathbf{6 a}, 6 \mathbf{6 b})$ which is missing from $N$ unsubstituted 4,5-annelated 1,3-oxazines, exhibiting the ions $\mathrm{C}_{6} \mathrm{H}_{7} \mathrm{~N}^{+\cdot}$ and $\mathrm{C}_{6} \mathrm{H}_{6} \mathrm{~N}^{+}$instead. ${ }^{29}$

\section{Comparison of regioisomeric pairs $1 \mathrm{a}-3 \mathrm{a}$ vs $\mathbf{1 b}-\mathbf{3 b}$}

The cyclization of the aminonaphtols used to synthesize these compounds are affected by different chemical stabilities of the unsubstituted or phenyl-substituted aminonaphthols. ${ }^{3}$ Since 1a-3a were synthesized from derivatives of (1- $\alpha$-aminobenzyl)-2-naphthol and $\mathbf{1} \mathbf{b}$-3b from derivatives of 1-aminomethyl-2-naphthol, ${ }^{4}$ the mass spectra of these regioisomeric pairs were expected to be very different. For $\mathbf{1 a}-\mathbf{3 a} \mathbf{M}^{+\bullet}$ was more abundant than for $\mathbf{1 b}-\mathbf{3 b}$. $[\mathrm{M}-\mathrm{OH}]^{+}$was observed only for 1a-3a. Exclusive ions for 1a-3a were $m / z 247, \mathrm{C}_{17} \mathrm{H}_{11} \mathrm{O}_{2}{ }^{+}$and $m / z 218$, $\mathrm{C}_{16} \mathrm{H}_{10} \mathrm{O}^{+\bullet}$. The ion $m / z 189, \mathrm{C}_{15} \mathrm{H}_{9}{ }^{+}$was also more abundant for 1a-3a than for $\mathbf{1 b}-\mathbf{b} \mathbf{b}$. For $\mathbf{1}, \mathbf{2} \mathbf{b}$ $\mathrm{C}_{8} \mathrm{H}_{7} \mathrm{NO}^{+\bullet}, m / z$ 133, was more abundant than for $\mathbf{1}, \mathbf{2 a}$ and the former also gave more abundant ions between $\mathrm{m} / z$ values 50 and 78 than the latter. In the case of $\mathbf{1}, \mathbf{2} \mathbf{b} \mathrm{C}_{8} \mathrm{H}_{7} \mathrm{NO}^{+\bullet}$ may be formed by a mechanism similar to that suggested for 1,3-benzoxazino-1,3-benzoxazines. ${ }^{16}$ 1a gave an abundant ion $\mathrm{C}_{17} \mathrm{H}_{11} \mathrm{O}^{+}$at $m / z 231$ which was missing from $\mathbf{1 b}$. For $\mathbf{2 b}$, 3a and $\mathbf{3 b} \mathrm{m} / z 231$ was the base peak, but for 2a its $R A$ was only $27 \%$. For $\mathbf{1 b}$ the ion $\mathrm{C}_{10} \mathrm{H}_{8}{ }^{+\bullet} \mathrm{m} / z 128$ was the base peak, but very weak for 1a. Compound 2a gave ions at $m / z 91\left(\mathrm{C}_{7} \mathrm{H}_{7}{ }^{+}\right)$and $m / z 118\left(\mathrm{C}_{8} \mathrm{H}_{8} \mathrm{~N}^{+}\right)$and 3a at $m / z 77\left(\mathrm{C}_{6} \mathrm{H}_{5}{ }^{+}\right), m / z 105\left(\mathrm{C}_{7} \mathrm{H}_{5} \mathrm{O}^{+}\right)$and $m / z 132\left(\mathrm{C}_{8} \mathrm{H}_{6} \mathrm{NO}^{+}\right)$, but these ions were missing from the spectra of $\mathbf{2 b}$ and $\mathbf{3 b}$, respectively.

${ }^{1} \mathrm{H} /{ }^{13} \mathrm{C}$ NMR analysis has been used to study the conformations of oxazine rings in the studied compounds. ${ }^{4}$ In $\mathbf{1 b}, \mathbf{2 b}, \mathbf{2 c}, \mathbf{2 d}, \mathbf{3 b}$ and the naphtho-bound oxazine ring prefers a 7,15twisted-chair conformation and, in 1a, 2a, 3a a 11,15b-twisted-chair conformation. In $\mathbf{1 b}, \mathbf{2 b}, \mathbf{2 c}$, 2d the benzo-fused oxazine ring prefers a 7a,12-twisted-chair conformation and in $1 \mathbf{a}$ and $2 \mathbf{a}$ a $11,15 \mathrm{~b}$-twisted-chair conformation. ${ }^{4}$ In $3 \mathbf{a}$ the benzo-fused oxazine ring prefers a 11,15b boat conformation. Based on calculations the benzo-fused oxazine ring in $\mathbf{3 b}$ is almost planar and the phenyl substituent is axial. ${ }^{4}$ These conformations possibly cause favored loss of $\mathrm{C}_{2} \mathrm{H}_{4} \mathrm{~N}^{\bullet}$ from the molecular ion of 1a leading to the product ion $\mathrm{C}_{17} \mathrm{H}_{11} \mathrm{O}_{2}{ }^{+}$, probably because in the $\mathrm{CH}_{2}$ groups the hydrogens are sterically less favored in $\mathbf{1 a}$ as compared to $\mathbf{1 b}$. Also the loss of $\mathrm{C}_{8} \mathrm{H}_{8} \mathrm{~N}^{\bullet}$ from 2a (i.e. $\mathrm{C}_{6} \mathrm{H}_{5} \mathrm{CHNCH}_{2}{ }^{\circ}$ ) corresponds to loss of $\mathrm{C}_{2} \mathrm{H}_{4} \mathrm{~N}^{*}$ from 1a, in both cases the product ion is $\mathrm{C}_{17} \mathrm{H}_{11} \mathrm{O}_{2}{ }^{+}$. The ion $\mathrm{C}_{2} \mathrm{H}_{4} \mathrm{~N}^{+}$is observed also for $\mathbf{1 a}$ and $\mathrm{C}_{8} \mathrm{H}_{8} \mathrm{~N}^{+}$for $\mathbf{2 a}$.

The formation of the $\mathrm{C}_{7} \mathrm{H}_{5} \mathrm{O}^{+}$ion is easier to explain for $3 a$, due to the tertiary $\alpha$-hydrogen vicinal to phenyl group which can migrate to the carbonyl oxygen. Also the boat conformation of benzo-fused oxazine ring in 3a makes the loss of $\mathrm{C}_{8} \mathrm{H}_{6} \mathrm{NO}^{\circ}$, i.e. the formation of the [M $\left.\mathrm{C}_{6} \mathrm{H}_{5} \mathrm{CHNCO}\right]^{+}$ion, $\mathrm{C}_{17} \mathrm{H}_{11} \mathrm{O}_{2}{ }^{+}$, possible.

\section{Conclusions}

Some of the fragmentations such as $\mathrm{OH}$-loss require ring-opening with hydrogen migration. In 7, $\mathbf{8 a}$ and $\mathbf{8 b}$ the carbonyl group oxygen participates in the loss of a hydroxyl group. Compounds 
derived from (1- $\alpha$-aminobenzyl)-2-naphthol were usually characterized by strong or medium strong ions at $m / z 231$ and 1-aminomethyl-2-naphthol derivatives by ions at $m / z 156$.

The fragmentations depend on the regiochemistry and geometry of the compounds studied. The formation of fragment ions $\left[\mathrm{M}-\mathrm{C}_{17} \mathrm{H}_{11} \mathrm{O}_{2}\right]^{+},\left[\mathrm{M}-\mathrm{C}_{17} \mathrm{H}_{12} \mathrm{O}\right]^{+\bullet}$ and $\left[\mathrm{M}-\mathrm{C}_{11} \mathrm{H}_{8} \mathrm{O}\right]^{+\bullet}$ occurred logically from the structures studied. However, these three ions were nearly missing from the spectra of derivatives $\mathbf{3 b}, \mathbf{4 a}, \mathbf{4 b}, \mathbf{6 b}, \mathbf{8 a}, \mathbf{8 b}$ with carbonyl or imino substituents; they favoured the ions $\mathrm{C}_{17} \mathrm{H}_{11} \mathrm{O}^{+}$or $\mathrm{C}_{11} \mathrm{H}_{8} \mathrm{O}^{+}$instead.

\section{Acknowledgements}

The authors wish to thank the Magnus Ehrnrooth Foundation for a grant to OM and PalomaaErikoski fund for a travel grant. The authors also wish to thank Ms Kirsti Wiinamäki for helping in recording the mass spectra.

\section{References}

1. Betti, M. Gazz. Chim. Ital. 1900, 30 II, 310.

2. Cardellicchio, C.; Ciccarella, G.; Naso, F.; Schingaro, E.; Scordari, F. Tetrahedron: Asymmetry 1998, 9, 3667 and references cited therein.

3. Szatmári, I.; Hetényi, A.; Lázár, L.; Fülöp, F. J. Heterocyclic Chem. 2004, 41, 367.

4. Heydenreich, M.; Koch, A.; Klod, S.; Szatmári, I.; Fülöp, F.; Kleinpeter, E. Tetrahedron 2006; 62: 11081.

5. Dong, Y.; Sun, J.; Wang, X.; Xu, X.; Cao, L.; Hu, Y. Tetrahedron: Asymmetry 2005, 15, 1667 and references cited therein.

6. Lu, J.; Xuenong, X.; Wang, C.; He, J.; Hu, Y.; Hu, H. Tetrahedron Lett. 2002, 43, 8367.

7. Ovcharenko, V.; Pihlaja, K.; Stájer, G. J. Am. Soc. Mass Spectrom. 2003, 14, 1049.

8. Pihlaja, K.; Martiskainen, O.; Stajer, G. Rapid Commun. Mass Spectrom. 2007, 21, 653.

9. Pihlaja, K.; Ovcharenko, V.; Stájer, G. J. Am. Soc. Mass. Spec. 1999, 10, 393.

10. Pihlaja, K.; Vainiotalo, P.; Fülöp, F.; Bernáth, G. Rapid Commun. Mass Spectrom. 1988, 2 , 229.

11. Pihlaja, K.; Lötjönen, L.; Fülöp, F.; Bernáth, G.; Vainiotalo, P. Rapid Commun. Mass Spectrom. 1990, 4, 279.

12. Vainiotalo, P.; Partanen, T.; Fülöp, F.; Bernáth, G.; Pihlaja, K, J. Am. Soc. Mass Spectrom. 1991, 2, 125.

13. Pihlaja, K.; Liukko-Sipi, S.; Fülöp, F.; Bernáth, G.; Vainiotalo, P. Rapid Commun. Mass Spectrom. 1991, 5, 230.

14. Fülöp, F.; Pihlaja, K.; Mattinen, J.; Bernáth, G, J. Org. Chem. 1987, 52, 3821.

15. Vainiotalo, P.; Fülop, F.; Pihlaja, K.; Org. Mass Spectrom. 1991, 26, 438. 
16. Joutsiniemi, K.; Vainiotalo, P.; Pihlaja, K.; Lázár, L.; Fülop, F.; Bernáth, G. Rapid Commun. Mass Spectrom. 1995, 9, 1035.

17. Juhász, M.; Fülöp, F.; Pihlaja, K. Rapid Commun. Mass Spectrom. 2007, 21, 3701.

18. Fülöp, F.; Pihlaja, K.; Mattinen, J.; Tetrahedron 1987, 43(8), 1863.

19. Fülöp, F.; Lázár, L.; Bernáth, G.; Sillanpää, R.; Pihlaja, K, Tetrahedron 1993, 49, 2115.

20. Vainiotalo, P.; Lehtelä, P-L.; Fülöp, F.; Bernáth, G.; Vuorilehto, L.; Pihlaja, K. Rapid Commun. Mass Spectrom. 1993, 7, 465.

21. Neuvonen, K.; Fülöp, F.; Neuvonen, H.; Koch, A.; Kleinpeter, E.; Pihlaja K. J. Org. Chem. 2001, 66, 4132.

22. Fülöp, F.; Pihlaja, K.; Mattinen, J.; Bernáth, G. Tetrahedron 1987, 43, 4565.

23. Szatmári, I.; Martinek, T.A.; Lázár, L.; Fülöp, F. Tetrahedron 2003, 59, 2877.

24. Szatmári, I.; Martinek, T.A.; Lázár, L.; Fülöp, F. Eur. J. Org. Chem. 2004, 2231.

25. Szatmári, I.; Martinek, T.A.; Lázár, L.; Koch, A.; Kleinpeter, E.; Neuvonen, K.; Fülöp, F. J. Org. Chem. 2004, 69, 3645.

26. Tomasulo, M.; Sortino, S.; White A. J. P.; Raymo F. M. J. Org. Chem. 2005, 70, 8180.

27. Tomasulo, M.; Sortino, S.; Raymo, F. M. J. Org. Chem. 2008, 73, 118.

28. Vainiotalo, P.; Fülop, F.; Stájer, G.; Bernáth, G.; Pihlaja, K. Acta Chem. Scand. 1990, 44, 165.

29. Oksman, P.; Pihlaja, K.; Fülöp, F.; Bernáth, G. Rapid Commun. Mass Spectrom. 1995, 9 , 615. 